\title{
Effects of Planetary Ball Milling on AlCoCrFeNi High Entropy Alloys prepared by Spark Plasma Sintering: Experiments and Molecular Dynamics Study
}

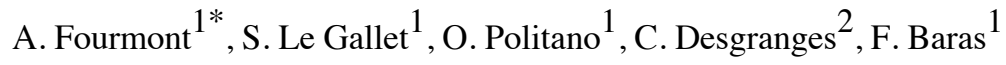 \\ 1. ICB, UMR 6303 CNRS-Université Bourgogne Franche-Comté, \\ 9 Av. Alain Savary, BP 47870, 21078 Dijon Cedex, France \\ 2. $\quad$ Safran Tech, Materials and Processes Department, \\ Rue des Jeunes Bois, Châteaufort, 78114 Magny-les-Hameaux, France \\ *Corresponding author: adrien.fourmont@u-bourgogne.fr
}

\begin{abstract}
The elaboration of High Entropy Alloys (HEA) was investigated by means of powder metallurgy. We studied the combination of mechanical activation and reactive sintering in the case of $\mathrm{AlCoCrFeNi}$. Elemental metallic powders were first processed by planetary ball milling over a long duration $(28 \mathrm{~h})$. The ratio $\mathrm{K}$ between the rotating speed of the sun wheel and the relative rotating speed of the grinding vials was set at 0.2 and 1 corresponding to "medium" energy milling. Given the particular hardness of chromium as compared to other elements, the effect of Cr powder size was investigated and optimized. In addition to experimental characterizations of milled powders, Molecular Dynamics simulations were carried out in order to assess the formation of solid solutions. The activated powders were then consolidated by Spark Plasma Sintering at $1000{ }^{\circ} \mathrm{C}$ and $1100{ }^{\circ} \mathrm{C}$. A nanostructured lamellar microstructure exhibiting the coexistence of the FCC and BCC phases was synthesized by this solid-state route. The sintered materials exhibited hardness of up to $670 \mathrm{HV}$. Our final results (i.e., after optimization of the milling and sintering parameters) suggest that mechanical activation combined with reactive sintering is an efficient route to elaborate dense HEA materials.
\end{abstract}

\section{Introduction}

High entropy alloys (HEA) contain five or more principal elements with each elemental concentration set between 5 at $\%$ and 35 at $\%$ [1]. They offer a wide range of properties and are a very promising field of study [2]. The $\mathrm{AlCoCrFeNi}$ composition has attracted much attention in recent years because it can easily be compared to 
common materials composed of similar elements (e.g., steels or M-Cr-Al alloys). These multi-component alloys are typically produced by arc-melting. When elaborated by means of this process, AlCoCrFeNi alloys exhibit a disordered $\mathrm{BCC}$ Cr-Fe rich phase and an ordered $\mathrm{BCC}$ Al-Ni rich phase [3-7]. Interesting mechanical properties [8] and encouraging oxidation resistance [9] have been reported. However, this type of elaboration, including a solidification step, may lead to the formation of dendrites and a deterioration of mechanical properties.

Other processes, such as Powder Metallurgy, have been studied and appear advantageous as compared to the common arc-melting process [10]. Indeed, a solid-state route enables industrial manufacturing and results in nanocrystalline materials with enhanced properties. Powder Metallurgy combines high-energy ball milling (HEBM) and consolidation processes such as Spark Plasma Sintering (SPS). High-energy ball milling has been extensively studied and is a rather versatile technique [11] that may induce either the mechanical activation of the green mixture or the complete transformation of the powder reactants. This latter process is usually referred to as Mechanical Alloying (MA), mechanosynthesis or mechanochemical synthesis. Most of the work on the elaboration of HEAs by powder metallurgy concerns the use of MA with a complete chemical reaction. In this case, the total consumption of the initial constituents can be verified, for instance, with XRD by noting the disappearance of elemental peaks. The synthesized powder is then consolidated by means of an appropriate technique. Concerning the $\mathrm{AlCoCrFeNi}$ alloys, the various investigations in the literature mainly concern MA. Ji et al. reported the formation of a BCC phase and an FCC phase with the combination of Mechanical Alloying (in a high-energy ball mill) and Spark Plasma Sintering [12], whereas Mohanty et al. found a more complex microstructure with precipitates of a tetragonal phase [13]. Yang et al. started from a glassy precursor in the MA+SPS route and obtained a microstructure similar to those obtained by arc-melting: an ordered BCC phase dispersed in a disordered BCC matrix [14]. Zhang et al. synthesized HEA using SPS only, starting from elemental powders without any HEBM step and reported the formation of a FCC phase and a duplex BCC structure [15]. Several investigations concern MA only. For instance, Vaidya et al. studied the influence of the sequential addition of elements during milling in Mechanical Alloying [16].

Very few variations have been explored. Colombini et al. considered the combination of simple mechanical activation of the green mixture followed by the microwave or field-assisted powder metallurgy route, but concluded that these techniques were not sufficiently efficient in attaining chemical homogeneity [17]. In the present work, we evaluate the use of mechanical activation (i.e., incomplete Mechanical Alloying) followed by a 
reactive sintering step. The mechanical activation enhances particle reactivity by creating defects and by increasing the number of interfaces between reactants [18]. The SPS treatment becomes crucial in producing HEA with the effective synthesis of activated powders. In addition, the versatility of HEBM provides new control parameters in order to achieve unexpected characteristics associated with the consolidation process, such as the non-equilibrium state. This specific elaboration route was found to lead to a unique HEA microstructure. Special attention was paid to the crystalline state of agglomerates after milling. In order to reach a better understanding of the milling results, molecular dynamics simulations (MD) were carried out, producing valuable information at the atomic scale. In addition, thermodynamic calculations provide an interesting counterpart to the experimental results.

\section{Methods}

\subsection{Experimental setup}

Elemental powders of $\mathrm{Al}, \mathrm{Co}, \mathrm{Cr}, \mathrm{Fe}$ and $\mathrm{Ni}$ of high purity (all $>99.2 \mathrm{wt} \%$, Alfa Aesar) were first blended with a TURBULA® mixer (WAB Turbula T2F) for 24 hours. Particle sizes were smaller than $45 \mu \mathrm{m}$ for $\mathrm{Al}, 2 \mu \mathrm{m}$ for $\mathrm{Co}, 75 \mu \mathrm{m}$ for $\mathrm{Cr}, 10 \mu \mathrm{m}$ for $\mathrm{Fe}$ and $15 \mu \mathrm{m}$ for Ni. Given that Chromium is the hardest material and has the largest particle size, a pre-milling of $\mathrm{Cr}$ powder was also undertaken to reduce its median for the volume distribution ( $\left.\mathrm{D}_{\mathrm{v}} 50\right)$ to $17 \mu \mathrm{m}$ before blending it with the other elemental powders. The pre-milling of $\mathrm{Cr}$ was performed in a high-energy ball mill (Fritsch Pulverisette 4). The pre-milling conditions were $250 \mathrm{rpm}$ for the rotating speed of the sun wheel, $250 \mathrm{rpm}$ for the relative speed of the grinding vial and $28 \mathrm{~h}$ duration with $2 \mathrm{~h}$ pauses every $7 \mathrm{~h}$. Pauses prevent the powder from agglomerating into large particles.

The blended powders in equimolar proportions were then processed by high-energy ball milling (Fritsch Pulverisette 4) with two sorts of duration used: $28 \mathrm{~h}$ processing without pausing and $28 \mathrm{~h}$ processing with $2 \mathrm{~h}$ pauses every $7 \mathrm{~h}$. Hardened steel grinding bowls and balls of $15 \mathrm{~mm}$ diameter were used. The ratio between the weight of the balls and the powders was set at $7: 1$. The ratio $\mathrm{K}=\omega / \Omega$ between the rotating speed of the sun wheel $\Omega$ and the relative speed of the grinding vial $\omega$ was set at 0.2 (milling M1) and 1 (milling M2). The different milling conditions are summarized in Table 1.

The powders were subsequently consolidated by means of Spark Plasma Sintering (FCT Systeme GmbH HPD 10) at $80 \mathrm{MPa}$ in a $10 \mathrm{~mm}$ inner-diameter graphite die. The milled powders obtained with initial $\mathrm{Cr}$ were sintered at 
$1000{ }^{\circ} \mathrm{C}$ whereas the milled powders obtained with pre-milled $\mathrm{Cr}$ were sintered at $1100{ }^{\circ} \mathrm{C}$. Two temperatures have been investigated because one aim of this work was to obtain the most interesting material in terms of microstructure. Indeed, two factors have been arbitrary chosen: the density of the sintered sample and the absence of weakening secondary phases. Increasing the temperature can improve the density and hinder the formation of secondary unwanted phases (for instance the tetragonal Cr-rich sigma phase is known to be stable at $1000^{\circ} \mathrm{C}$ but not at $\left.1100^{\circ} \mathrm{C}[9]\right)$. These two temperatures are quite high compared to sintering temperatures for Mechanically Alloyed powders. Indeed, diffusion may need to be favored for the alloying to occur completely during the reactive sintering. The same heating rate of $50^{\circ} \mathrm{C} / \mathrm{min}$ was used in both cases. No dwell time was applied in order to avoid grain growth and to preserve the nanostructure of the material. Sintering was carried out in a vacuum. Grinding bowls and SPS dies were filled in a glove box under argon atmosphere in order to avoid possible oxygen contamination. The sintered samples were $5 \mathrm{~mm}$ thick discs with a radius of $1 \mathrm{~cm}$. The sintering conditions are reported in Table 1.

The powder size distribution was measured by laser granulometry (Malvern 2000). The powders and the sintered samples were analyzed using X-Ray Diffraction with $\mathrm{Cu} \mathrm{K} \alpha$ radiation (XRD, Siemens D5000), scanning electron microscopy and electron backscatter diffraction (SEM and EBSD, JEOL JSM 7600F), inductively coupled plasma atomic emission spectrometry (ICP-AES, Thermo Electron iCAP 7400) and X-Ray Fluorescence (XRF, Brucker S8 Tiger). The upper surfaces of the sintered samples were observed after polishing. The density of all the sintered samples was determined via hydro-static weighing using Archimede's method. Relative density is the ratio between the sample density and the theoretical density, as determined by the mixing law $\left(6978 \mathrm{~kg} / \mathrm{m}^{3}\right)$. Hardness was measured using Vickers indenters (ZwickRoell ZHV $\mu$ ) with a load of $100 \mathrm{~g}$ and a 10 s dwell time. Certain hardness markers were precisely observed using SEM to ensure the accuracy of the hardness measurement. 
Table 1: Milling and sintering conditions. Milling $M 1$ refers to $K=0.2$ and milling $M 2$ to $K=1$. Batch conditions are denoted Mi-j where $i$ corresponds to the milling mode ( $i=1$ or 2 ), $j$ corresponds to the characteristics of the $\mathrm{Cr}$ powder $(\mathrm{j}=\mathrm{CR}$ is for the large $75 \mu \mathrm{m}$ powder and $\mathrm{j}=\mathrm{cr}$ for the pre-milled powder). Milling duration was usually $4 \times 7 \mathrm{~h}$ milling with $2 \mathrm{~h}$ pauses. A long milling duration (28h) without pauses is specified by " $L$ " for long duration.

\begin{tabular}{|c|c|c|c|c|c|c|c|c|c|}
\hline \multirow[b]{2}{*}{ Cr Powder } & \multicolumn{4}{|c|}{ Milling } & \multirow[b]{2}{*}{ Batch } & \multicolumn{4}{|c|}{ Sintering } \\
\hline & Mode & $\begin{array}{c}\mathbf{\Omega} \\
(\mathbf{r p m})\end{array}$ & $\begin{array}{c}\omega \\
(\mathbf{r p m})\end{array}$ & $\begin{array}{c}\text { Duration } \\
\text { (hours) }\end{array}$ & & $\begin{array}{c}\mathbf{T} \\
\left({ }^{\circ} \mathbf{C}\right)\end{array}$ & $\begin{array}{l}\text { Heating } \\
\text { rate } \\
\left({ }^{\circ} \mathrm{C} / \mathrm{min}\right)\end{array}$ & $\begin{array}{c}\text { Dwell } \\
\text { time }\end{array}$ & $\begin{array}{c}\mathbf{P} \\
(\mathbf{M P a})\end{array}$ \\
\hline \multirow{2}{*}{ initial } & \multirow{3}{*}{ M1 } & \multirow{3}{*}{250} & \multirow{3}{*}{-50} & 28 & M1-CR-L & 1000 & \multirow{6}{*}{50} & \multirow{6}{*}{-} & \multirow{6}{*}{80} \\
\hline & & & & \multirow{2}{*}{$4 \times 7$} & M1-CR & 1000 & & & \\
\hline pre-milled & & & & & M1-cr & 1100 & & & \\
\hline intitol & \multirow{3}{*}{ M2 } & \multirow{3}{*}{250} & \multirow{3}{*}{-250} & 28 & M2-CR-L & \multirow{2}{*}{1000} & & & \\
\hline milra & & & & \multirow{2}{*}{$4 \times 7$} & M2-CR & & & & \\
\hline pre-milled & & & & & M2-cr & 1100 & & & \\
\hline
\end{tabular}

\subsection{Modeling approach}

In order to better understand the milling process and shed some light on the experimental results, molecular dynamics (MD) simulations were carried out using the LAMMPS code [19]. The main goal of these simulations was to evaluate the evolution of the lattice parameter for different structures during the formation of solid solutions by mechanical alloying. Interatomic interactions are described by an Embedded Atom potential (EAM) developed by Zhou for several pure metals, especially for Al, Co Fe, Ni [20] and by Lin et al. for Cr [21]. The cross-potential for multi-component systems is obtained by the weighted averages of elemental components [22]. The mechanical properties of a wide range of metallic elements are correctly described, including the pure metals of interest here. Two types of simulation were performed. The first type aims at mimicking the chemical mixing induced by plastic deformation [23]. The second one creates a random solid solution of different elements.

In the first type of simulation, a polycrystalline structure is generated for different, nearly equimolar ternary alloys.

The structure contains 18 grains of average diameter equal to $40 \AA$ created by using Voronoi tessellation [24]. Each grain is initially composed of one of the pure elements. The total number of atoms and the compositions are summarized in Table 2. The combinations of 3 elements proposed are justified by the XRD results presented further in this work (paragraph 3.1). An energy minimization was first carried out under periodic boundary conditions to allow atoms located at grain boundaries to rearrange. A thermalization step was then performed: at $100 \mathrm{~K}$ for $50 \mathrm{ps}$ in the NVT ensemble and a second run at the same temperature and zero pressure for $50 \mathrm{ps}$ in NPT ensemble. As underlined in [18], the temperature was chosen below ambient temperature to avoid any thermally 
activated diffusion. Afterwards, 100 cyclical compressions along the 3 directions of the simulation box were applied in the NPT ensemble. One compression cycle lasted 150 ps and consisted of 3 successive deformations of 50 ps along $\mathrm{X}, \mathrm{Y}$ and $\mathrm{Z}$. To perform the deformation along the considered direction (e.g. X), the box size was rescaled 500 times (i.e. every $0.1 \mathrm{ps)}$ ) to gradually reach a final strain of $25 \%$ after $50 \mathrm{ps.} \mathrm{After} \mathrm{each} \mathrm{rescaling,} \mathrm{the}$ atomic positions were remapped into the new box in the appropriate manner. The two other dimensions of the box (e.g. Y and Z) were allowed to move independently (i.e., anisotropic NPT) in order to maintain zero pressure along these directions. After $50 \mathrm{ps,} \mathrm{the} \mathrm{same} \mathrm{procedure} \mathrm{was} \mathrm{repeated} \mathrm{along} \mathrm{Y}$ and $\mathrm{Z}$ to complete a full compression cycle. In all, the total simulation run lasted 15 ns (i.e., 100 cycles x 3 directions x 50 ps). The atomic positions were relaxed before measuring relevant indicators. It was verified that this minimization only reduced the noise on the indicators but did not change the atomic configuration obtained by the deformation procedure. These simulations aimed to describe the mixing driven by plastic deformation (PDM) that occurs during milling.

For the second type of simulation, an initial bcc-Fe or - $\mathrm{Cr}$ (or fcc-Ni) structure with the appropriate lattice parameter was constructed. Then, Al and Co atoms were randomly substituted. The simulation box (anisotropic volume change) and the atomic positions were then relaxed and relevant indicators measured. In this case, the mixing is due to a random substitution (RSM) procedure.

The final microstructure was analyzed using relevant indicators:

- The crystal structure was assessed by means of adaptive CNA (common neighbor analysis by Stukowski et al. [25]), which compares the local coordination of all atoms to perfect crystalline structures (bcc, fcc, hcp, ico) and assigns them the structure which best fits. The specificity of the adaptative CNA is that it determines a local cut off radius for each atom. It is particularly useful in the case of a simulation exhibiting different structures and consisting of several elements.

- The lattice parameters were calculated using well defined peaks in the Radial Distribution Function. This function was averaged over all atoms of the simulation box.

- The chemical mixing was monitored by the Micro-Chemical Inhomogeneity parameter (MCI), $\zeta$, which is useful to characterize a mixture with $\mathrm{N}$ types of atoms (particularly when $\mathrm{N}>2$ ) [26]. The average number of neighbors around an atom of type $i, Z_{i}$, is the sum of the average number of $i-j$ nearest neighbors $Z_{i j}$

$$
Z_{i}=\sum_{j} Z_{i j}
$$


Next, the ratio of similar and dissimilar nearest neighbors is introduced:

$$
\begin{aligned}
& p_{i i}=Z_{i i} / Z_{i} \\
& p_{i j}={ }^{i j} / Z_{i}
\end{aligned}
$$

and a local parameter is defined

$$
\zeta_{i}=p_{i i}-\frac{1}{N-1} \sum_{j} p_{i j}
$$

whose summation gives the MCI parameter

$$
\zeta=\sum_{i} \zeta_{i} \cdot c_{i}
$$

For an equimolar mixture, $\zeta$ ranges from 1 (perfectly segregated) to 0 (randomly mixed $=$ the average local composition being the same as the global one). In the case of a non-equimolar mixture, the ideal value of the mixed state can be calculated.

Table 2: Characteristics of the simulated systems.

\begin{tabular}{|c|c|c|c|c|}
\hline System & $\mathrm{Al}$ (at. \%) & $\mathrm{Co}($ at. \%) & $\mathrm{Ni} / \mathrm{Fe} / \mathrm{Cr}$ (at. \%) & Number of atoms \\
\hline $\mathrm{AlCoNi}$ & 24.7 & 37.2 & 38.1 & 64693 \\
\hline $\mathrm{AlCoFe}$ & 25.4 & 38.3 & 36.2 & 62800 \\
\hline $\mathrm{AlCoCr}$ & 25.4 & 38.3 & 36.2 & 62800 \\
\hline
\end{tabular}
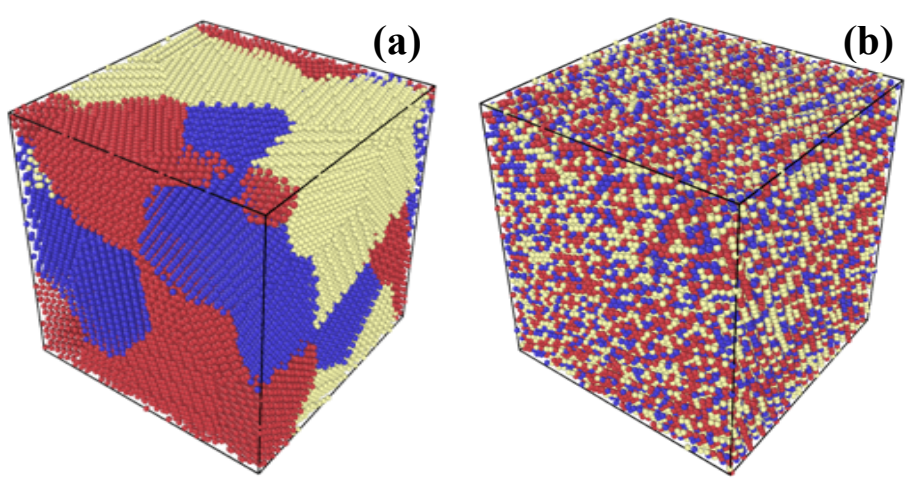

Figure 1: (a) Initial polycrystalline system with 18 grains of average diameter $40 \AA$ and (b) final microstructure after mixing driven by plastic deformation simulation. The system shown is $\mathrm{Al}-\mathrm{Co}-\mathrm{Fe}$ ( $\mathrm{Al}$ in red, $\mathrm{Co}$ in blue and $\mathrm{Fe}$ in yellow). 


\section{Results and discussion}

\subsection{Milling}

Cross sections of representative agglomerates of each milling mode are displayed in Fig. 2. After milling M1, the agglomerates exhibit a granular microstructure composed of almost pure elemental particles. Each contrast corresponds to an almost pure element; the black area is Al. After milling M2, it becomes nearly impossible to precisely identify each elemental zone. The microstructure becomes lamellar in most agglomerates. The characteristic dimension of these lamellar zones is less than $100 \mathrm{~nm}$. As indicated by the several remaining contrasts in Back Scattering Electron (BSE) mode, complete Mechanical Alloying has not been achieved, we are still at the stage of mechanical activation.
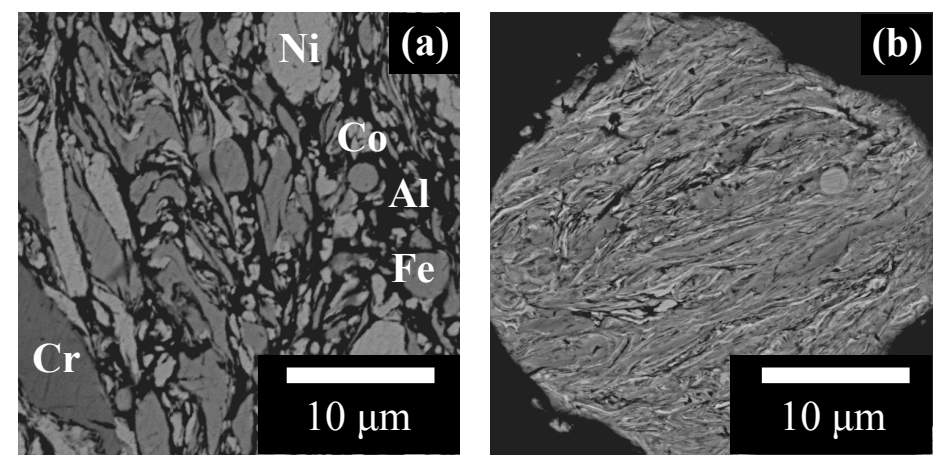

Figure 2: SEM images (BSE mode) of cross sections of (a) M1-CR-L and (b) M2-CR-L powders.

The compositions of the milled powders (M1-CR-L and M2-CR-L) were characterized by ICP and compared to that of a blended powder (Table 3). There is no evidence of contamination by Fe from the grinding media or of any loss of elements that could have preferentially adhered to the vials during the milling step. The analysis shows an increase in chromium, probably due to the poor chemical homogeneity of the M1-milled powder.

Table 3: ICP results on blended and milled powders.

\begin{tabular}{|l|c|c|c|c|c|}
\hline at. \% & $\mathrm{Al}$ & $\mathrm{Co}$ & $\mathrm{Cr}$ & $\mathrm{Fe}$ & $\mathrm{Ni}$ \\
\hline blended powder & 19.5 & 19.2 & 21.4 & 19.7 & 20.2 \\
\hline M1-CR-L & 17.5 & 17.8 & 30.3 & 17.7 & 16.7 \\
\hline M2-CR-L & 19.6 & 19.9 & 21.2 & 19.8 & 19.5 \\
\hline
\end{tabular}


X-Ray Diffraction patterns of the powders are shown in Fig. 3-a. For the blended powder, all elemental peaks are identified. However, an XRD quantitative analysis (Rietveld analysis) shows that chromium is underestimated. This observation can likely be attributed to the peculiar crystalline state with nanosized crystallites and a possible texture within particles, already existing in the commercial $\mathrm{Cr}$ powder. Indeed, the Rietveld analysis indicates that the average crystallites size is $76 \mathrm{~nm}$ and that the standard relative intensity between peaks is not respected.

After milling, the intensity of the $\mathrm{Al}$ and Co peaks decreased compared to the other phases. There is a strong decrease in the case of the M1 milling, whereas it is a complete disappearance in the other case. In both cases, there might be a slight amorphous halo. There are no significant shifts in the other peak positions. The lattice parameter of the Ni/FCC phase remains around $3.525 \AA$ and those of the $\mathrm{Fe}$ and $\mathrm{Cr} / \mathrm{BCC}$ phases around $2.87 \AA$. However, the FCC peaks become asymmetrical after M2 milling (Fig. 3-b). At this level of analysis, the size of the chromium particle (commercial or pre-milled) and defects density (in the initial powder or introduced by the pre-milling) do not significantly affect the milling mechanism.

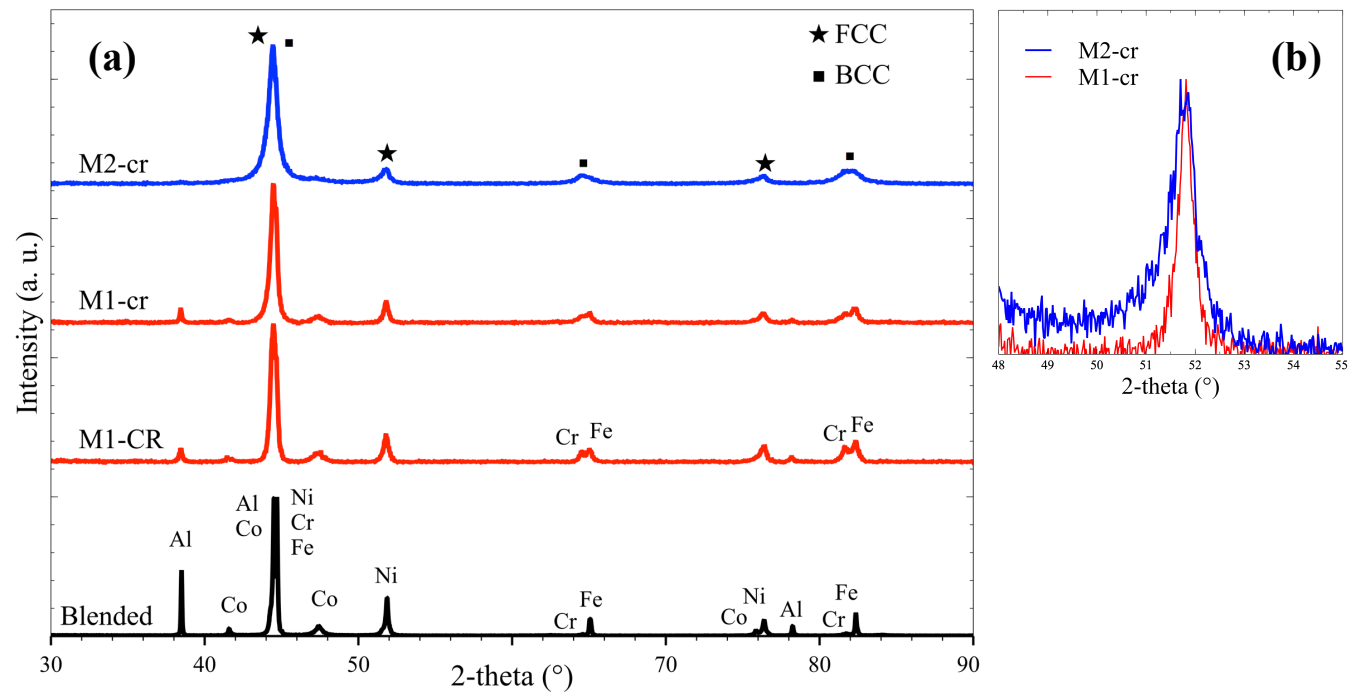

Figure 3: (a) X-ray diffraction patterns of milled powders (M1-CR, M1-cr, M2-cr and blended powders). The intensities are normalized on the main BCC peak. (b) Close up view of the normalized (200) peak of the FCC to show the asymmetry.

At this point, it is difficult to claim that solid solutions are forming during the milling. Indeed, the disappearance of the Co and especially the Al peaks associated with the non-evolution of the lattice parameters are two clues pointing to two different conclusions. The $\mathrm{Al}$ atomic radius $(143 \mathrm{pm})$ is larger than the others $(\sim 125 \mathrm{pm}$ on average [27]). Because of the larger size of Al atoms, Vegard's law predicts that substituting $\mathrm{Al}$ and Co atoms into Ni, $\mathrm{Fe}$ and/or Cr matrices would cause an increase in the lattice parameter. Since we observed both the disappearance of $\mathrm{Al}$ and Co peaks in XRD patterns and the persistence of two phases, we studied the case of 3-element systems: 
Al-Co-Ni for the FCC-phase and Al-Co-Fe and/or Al-Co-Cr for the BCC-phase. Vegard's law gives a lattice parameter of $3.66 \AA$ for a 3-element FCC-system of composition $\mathrm{Al}_{24.7} \mathrm{Co}_{37.2} \mathrm{Ni}_{38.1}$ and $2.98 \AA$ for a 3-element BCC-system of composition $\mathrm{Al}_{25.4} \mathrm{Co}_{38.3} \mathrm{Fe}_{36.2}$ or $\mathrm{Al}_{25.4} \mathrm{Co}_{38.3} \mathrm{Cr}_{36.2}$, as compared to $3.52 \AA$ for pure $\mathrm{Ni}, 2.87 \AA$ for pure Fe, and $2.88 \AA$ for pure $\mathrm{Cr}$.

In order to understand the evolution of the lattice parameter during the milling, MD simulations were performed. The exact composition of each system is of lesser importance since the main question here is binary: Does the lattice parameter really increase with substitution in these cases, as Vegard's law indicates? The MD results are summarized in Table 4. Note that the PDM procedure was carried out for the AlCoCr system; however, it did not form a solid solution but rather a bcc-Cr and an amorphous phase. Indeed, $\mathrm{Cr}$ is known to be imperfectly fitted by an EAM and its properties are poorly reproduced in MD [28]. In addition, high $\mathrm{Cr}$ hardness might prevent $\mathrm{Al}$ and Co substitution into its structure.

Table 4: Measured indicators (lattice parameters in $\AA$, at. \% of crystal structure and Microchemical Inhomogeneity) obtained by Molecular Dynamics simulations following the PDM or RSM procedures.

\begin{tabular}{|l|l|l|l|l|}
\hline System & Type of MD simulations & Lattice Parameter & \% FCC or BCC & MCI parameter (Ideal mixing) \\
\hline AlCoNi & PDM & 3.646 & 55 & $0.05(0.02)$ \\
\cline { 2 - 5 } & RSM & 3.633 & 100 & $-0.01(0.02)$ \\
\hline \multirow{2}{*}{$\mathrm{AlCoFe}$} & PDM & 2.877 & 73 & $0.02(0.01)$ \\
\cline { 2 - 5 } & RSM & 2.895 & 100 & $-0.01(0.01)$ \\
\hline $\mathrm{AlCoCr}$ & RSM & 2.884 & 100 & $-0.01(0.01)$ \\
\hline
\end{tabular}

First, the values of the MCI parameter $\zeta$ (eq. (5)) indicate that in both kinds of simulation, local mixing is achieved (the initial $\zeta$-values for non-mixed polycrystalline systems are $\sim 0.9$ ). Thus there are no major segregations in the final systems: the solid solutions have indeed been formed. Secondly, the amounts of FCC and BCC in the final system after the PDM simulation show that the main part of the system is indeed crystallized (the remaining part being amorphous). The most interesting values of the MD simulations are the lattice parameters. For the FCC $\mathrm{Al}_{24.7} \mathrm{Co}_{37.2 \mathrm{Ni}} \mathrm{Ni}_{31} \mathrm{system}$, the lattice parameter increases significantly from the pure $\mathrm{Ni}$ to the solid solution $(+3.5 \%)$. However, for the BCC structures, there is only a small increase in the case of the simulation $(1 \%)$. The evolution of the BCC lattice parameter seems not to obey Vegard's law.

It can be concluded that, in our case, an FCC solid solution is only beginning to form since the experimental FCC lattice parameter is not really increasing but the peaks are becoming asymmetrical (Fig. 3b). The Al and Co atoms 
may have already partly substituted for the $\mathrm{Fe}$ and/or $\mathrm{Cr}$ atoms in the $\mathrm{BCC}$ structure since the lattice parameter does not seem to evolve much with the substitution ratio. Similar observations have been reported in the literature. After a milling step more powerful than the one used in this work, Mohanty et al. observed a lattice parameter of $3.56 \pm 0.02 \AA(3.52 \AA$ for pure Ni) for the FCC-phase and $2.89 \pm 0.05 \AA(2.87 \AA / 2.88 \AA)$ for the BBC-phase [13]. Ji et al. observed a BCC lattice parameter of $2.88 \AA ̊$ after a milling step powerful enough to reach complete Mechanical Alloying [12]. A small portion of the milled powder may also be constituted by an amorphous phase; indeed, Portnoi et al. reported the formation of an amorphous phase after $2 \mathrm{~h}$ milling only for the AlCoCrFeNiTi alloy [29].

The milled powders were also analyzed by particle size analysis (laser granulometry). The results are displayed in Table 5. Particle size distribution is influenced by the milling conditions. The M2 milling results in smaller agglomerates. $\mathrm{D}_{\mathrm{v}} 50$ is 4.8 times smaller than those obtained with $\mathrm{M} 1$ milling. Introducing pauses leads to thinner particles in M1 and M2. D 50 is 2.3 times lower than that obtained without pauses. It may be argued that a higher temperature is reached over a longer duration without pauses. Aluminum particles particularly could act as a binder in conditions of higher temperature. Chromium pre-milling does not affect either the $\mathrm{D}_{\mathrm{v}} 50$ or the agglomerates size distribution of the final 5-element batches. Hence, chromium pre-milling solely impacts the microstructure of the agglomerates.

Table 5: Particle size distribution of the milled powders.

\begin{tabular}{|c|c|c|c|}
\hline Batch & $\mathrm{D}_{\mathrm{v}} 10(\mu \mathrm{m})$ & $\mathrm{D}_{\mathrm{v}} 50(\mu \mathrm{m})$ & $\mathrm{D}_{\mathrm{v}} 90(\mu \mathrm{m})$ \\
\hline M1-CR-L & 130 & 389 & 625 \\
\hline M1-CR & 49 & 166 & 533 \\
\hline M1-cr & 39 & 159 & 528 \\
\hline M2-cr & 11 & 33 & 98 \\
\hline
\end{tabular}

At this stage, it appears that milling conditions affect milled powders in terms of their morphology, their microstructure and the arrangement at the microscopic scale. In order to quantify the milling efficiency, we adopted a very common, although questionable, point of view based on kinematic models [21,22]. Burgio's model gives the shock energy and power as a function of milling parameters (see Table 6) [30]. According to this approach, collision power is the key factor impacting the final crystallinity and milling progress [31]. Millings M1 and M2 used in this work are lower in energy that those commonly found in the literature, which often lead to total disappearance of the FCC phase during the milling step [12,32]. This estimation suggests that neither of our two milling modes do actually achieve complete Mechanical Alloying. Although these simple kinematic models are 
very popular, different works based on direct visualization of ball motion in the vials [33] and discrete element methods [34] demonstrate their limitations in accurately describing milling regimes, especially in terms of the relative influence of collision events over friction.

Table 6: Calculated quantities (energy by hit and by ball, power (W) by hit and by ball, total energy of the milling by ball) using Burgio's model. Note that these quantities are normalized 'by ball' in order to compare several millings with different numbers of balls.

\begin{tabular}{|l|c|c|c|c|c|}
\hline Routes & $\mathrm{mJ} / \mathrm{hit} / \mathrm{g}$ & $\mathrm{mW} / \mathrm{hit} / \mathrm{g}$ & $\mathrm{E}_{\mathrm{tot}}(\mathrm{kJ} / \mathrm{g})$ & $\mathrm{K}$ ratio & Centrifugal acceleration \\
\hline M1 & 3 & 47 & 5 & -0.20 & $9 \mathrm{~g}$ \\
\hline M2 & 8 & 109 & 11 & -1 & $4 \mathrm{~g}$ \\
\hline $\begin{array}{l}\text { Moravcik [32] for } \\
\text { AlCoCrFeNiTi0.5 }\end{array}$ & 16 & 221 & 19 & -1.82 & $9 \mathrm{~g}$ \\
\hline Mohanty [13] & 12 & 130 & 12 & -2 & \\
\hline
\end{tabular}




\subsection{Sintering}

The different samples presented in Table 1 were sintered using SPS. XRD diffraction patterns are depicted in Fig. 4 for M1-CR-L and M2-CR-L at $1000^{\circ} \mathrm{C}$ (commercial Cr with low energetic milling M1 and higher energetic milling M2 of 28h duration) and M1-cr and $\mathrm{M} 2$-cr at $1100^{\circ} \mathrm{C}$ (pre-milled $\mathrm{Cr}$ low energetic milling $\mathrm{M} 1$ and higher energetic milling M2 of 4x7h duration with pauses). This characterization allows us to follow the modifications due to sintering as compared to milling (see Fig. 3).

The main observations are as follows:

- An ordered BCC phase (B2) is formed. Cieslak et al. claim that XRD characterization does not offer the opportunity to prove the existence of a single B2 phase or an ordered B2 and a disordered A2 phase [35]. However, by considering either one $\mathrm{BCC}$ phase (B2) or two $\mathrm{BCC}$ phases (B2+A2), the Rietveld analysis presents a better goodness of fit for the first hypothesis of a single B2 phase.

- The FCC peaks clearly shift to lower angles: the FCC phase is not pure Ni at all. For the M1-cr and M2cr samples, the lattice parameters were calculated using a Rietveld analysis. The FCC lattice parameter is found to be $3.595 \AA$ in both samples. Here, the FCC lattice parameters of M1-cr and M2-cr have increased, meaning that the FCC solid solution was formed during sintering.

- The small peaks around the two main peaks correspond to a tetragonal sigma phase. The sigma phase has often been observed by other researchers, both in powder metallurgy [13] and casting [5]. The sigma phase is present at $1000^{\circ} \mathrm{C}$ when the elemental powders are M2-milled. There remain only traces of this phase at $1100^{\circ} \mathrm{C}$ for M1-milled powders. Here, the presence of the sigma phase depends on the combination of the milling conditions, the sintering temperature and $\mathrm{Cr}$ particles size.

Besides phase characterization, the Rietveld analysis provides interesting information about the structure of the sintered samples. The M1-cr and M2-cr samples, sintered at $1100^{\circ} \mathrm{C}$, are nanostructured:

- $\quad$ M1-cr: BCC phase crystallites size $85 \mathrm{~nm}$, FCC phase crystallites size $140 \mathrm{~nm}$;

- $\quad$ M2-cr: BCC 125 nm, FCC $100 \mathrm{~nm}$. 


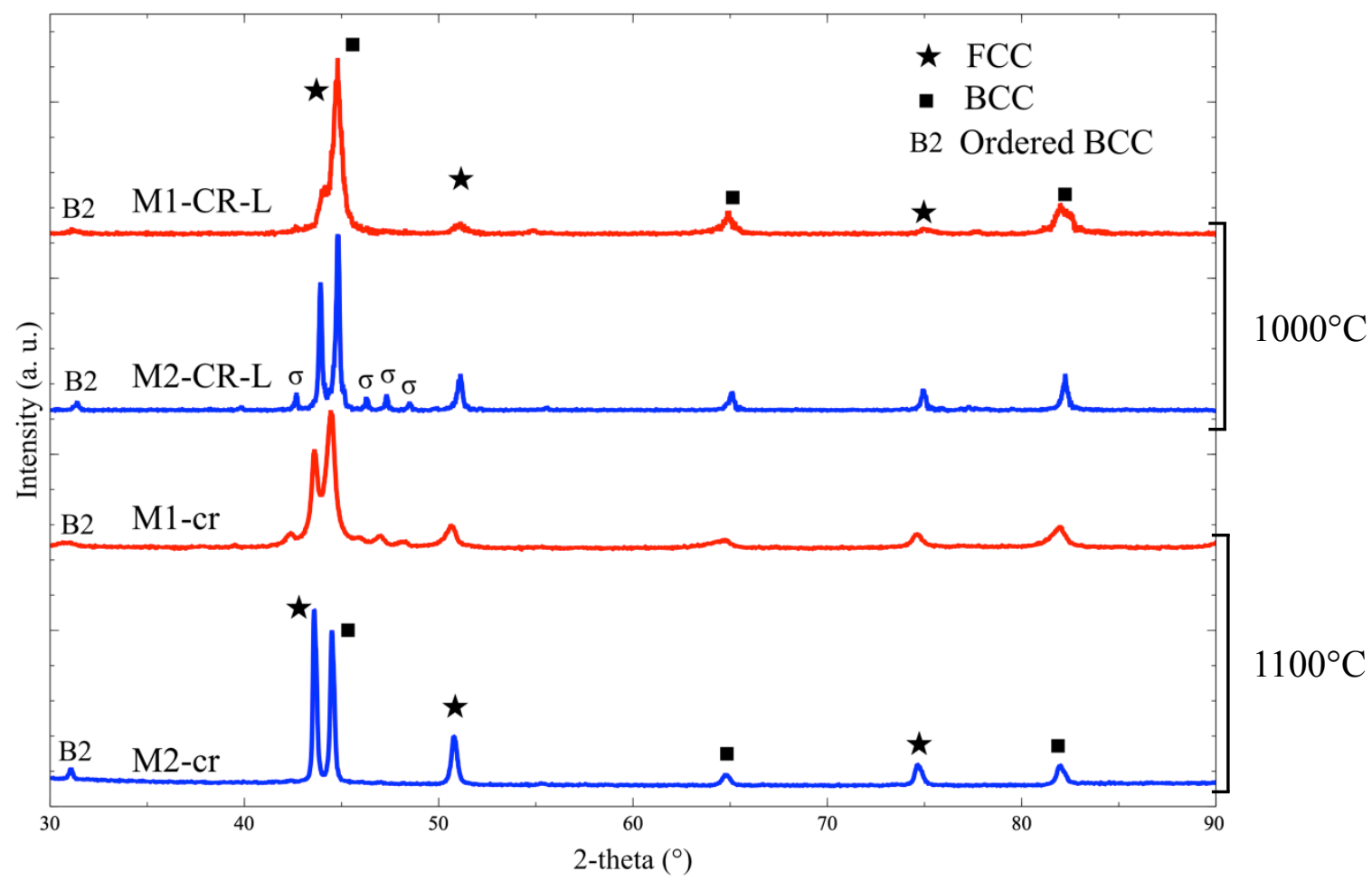

Figure 4: X-ray diffraction patterns of M1-CR-L, M2-CR-L (sintered at $1000^{\circ} \mathrm{C}$ ) $\mathrm{M} 1-\mathrm{cr}, \mathrm{M} 2-\mathrm{cr}\left(\operatorname{sintered}\right.$ at $1100^{\circ} \mathrm{C}$ ) samples. The intensities are normalized on the main BCC peak.

M1-CR-L
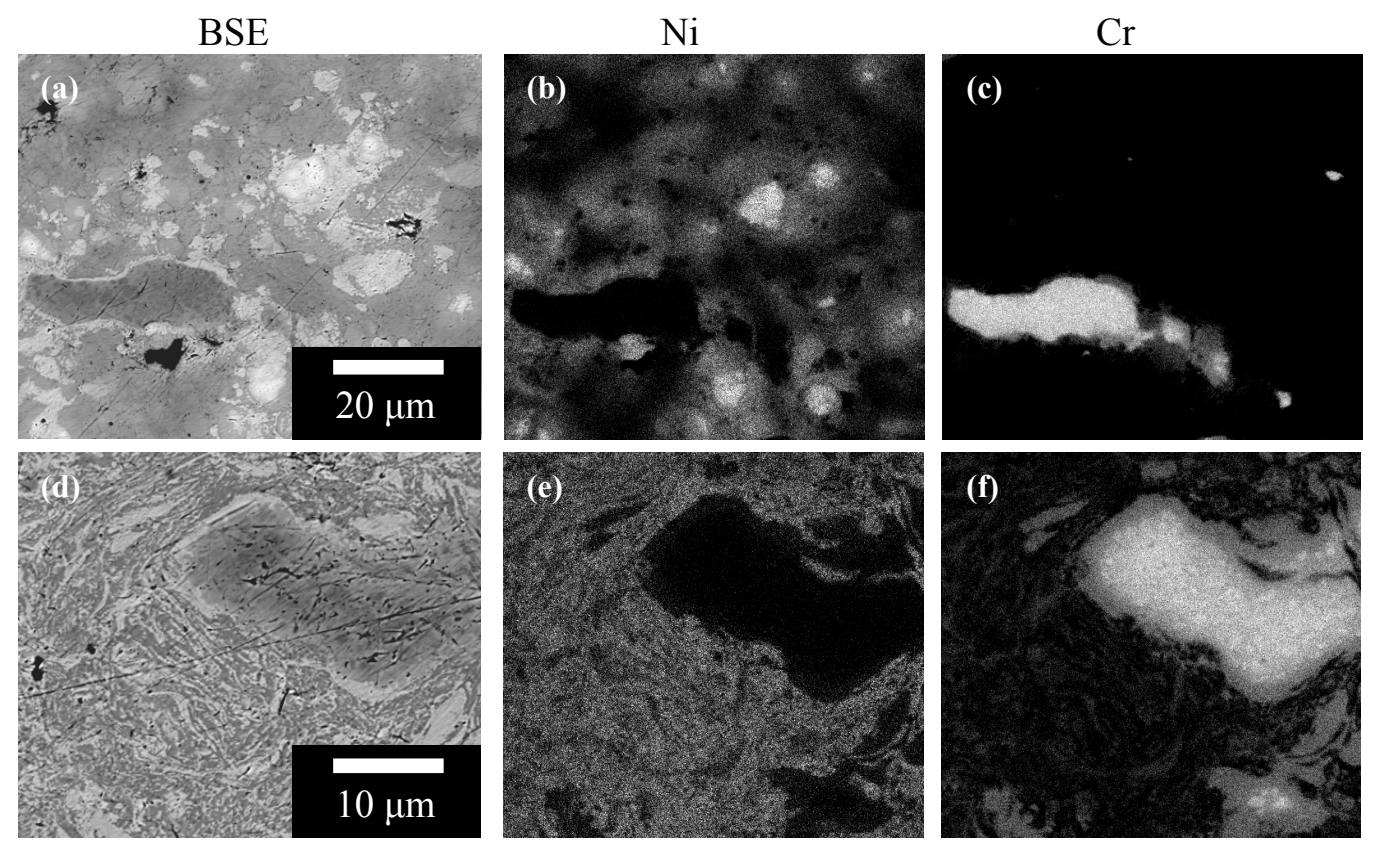

Figure 5: SEM image (BSE mode) (a), Ni distribution (b) and Cr distribution (c) of the samples M1-CR-L sintered at $1000^{\circ} \mathrm{C}$. SEM image (BSE mode) (d), Ni distribution (e) and $\mathrm{Cr}$ distribution (f) of the samples M2-CR-L sintered at $1000^{\circ} \mathrm{C}$. 
The microstructure of sintered samples was investigated by means of usual SEM characterization. Figure 5 shows the SEM images of samples sintered at $1000{ }^{\circ} \mathrm{C}$ with commercial chromium. It is evident that milling conditions affect the microstructure. An energetic milling favors a lamellar structure (Fig. 5a and Fig. 5d). Let us note that the M1-sample microstructure remains heterogeneous (Fig. 5a) as compared to agglomerates (Fig. 2a). In this case, sintering does not drastically modify the distribution of elements observed after the milling step. EDX cartography demonstrates that elemental distribution is far from being homogenous (Fig. 5b 5c 5e 5f). Highly concentrated Ni zones are still present in the M1-sample, whereas $\mathrm{Ni}$ is rather well mixed in the M2 sample. For both millings, initial chromium grains remain practically intact and $\mathrm{Cr}$ barely diffuses into the rest of the sample.

As shown in Fig. 6, sintering results in a better chemical homogeneity, when the temperature is increased to
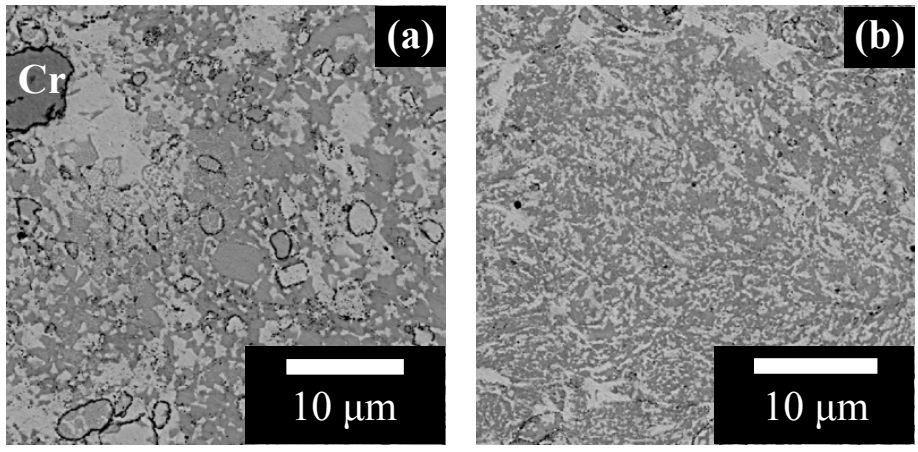

Figure 4: SEM images (BSE mode) of microstructure of M1-cr sintered at $1100{ }^{\circ} \mathrm{C}$ (a) and $\mathrm{M} 2$-cr samples sintered at $1100{ }^{\circ} \mathrm{C}(\mathrm{b})$.

$1100{ }^{\circ} \mathrm{C}$ and chromium is pre-milled. M1-samples consist mainly of two phases and chromium particles (Fig. 6a). There are also black dots around the grains: these have not yet been precisely analyzed, but they must be carbides and/or oxides (mainly $\mathrm{Cr}_{x} \mathrm{C}_{y}$ [36] and $\mathrm{Al}_{2} \mathrm{O}_{3}$ [32]). EDX mapping confirms this hypothesis. It also suggests the presence of chromium oxides (see Supplementary Material, Fig. A1). The microstructure of M2-samples is still lamellar and exhibits two to three contrasts in the BSE mode.

Finally, the modification of two parameters (temperature and chromium initial size) leads to a more interesting material. Indeed, compared to M1-samples sintered at $1000{ }^{\circ} \mathrm{C}$, the distribution of the two main phases is much thinner in the M2-samples sintered at $1100^{\circ} \mathrm{C}$. Hence, M2-cr parameters genuinely lead to unique and interesting HEAs. 
Table 7: Compositions (in at.\%) of the different phases measured from EDX.

\begin{tabular}{|l|l|l|l|l|l|l|}
\hline Samples & SEM Contrast & $\mathrm{Al}$ & $\mathrm{Co}$ & $\mathrm{Cr}$ & $\mathrm{Fe}$ & $\mathrm{Ni}$ \\
\hline \multirow{2}{*}{ M1-cr } & Light-grey phase & 9.6 & 23 & 23.7 & 25.7 & 18 \\
\cline { 2 - 8 } & Medium-grey phase & 25 & 19 & 13 & 17 & 26 \\
\hline \multirow{2}{*}{ M2-cr } & Light-grey phase & 8 & 25 & 25.7 & 25.7 & 15.6 \\
\cline { 2 - 7 } & Medium-grey phase & 27.5 & 17.5 & 16 & 15 & 24 \\
\hline
\end{tabular}

The composition associated with each observed BSE phase was determined by EDX analysis (see Table 7):

- $\quad$ The light-grey contrast corresponds to an Al-Ni poor and Fe-Cr-Co rich composition.

- The medium-grey contrast corresponds to an Al-Ni rich and Fe-Cr-Co poor composition.

The $\sigma$ phase $\left(\simeq \mathrm{Co} 0.25 \mathrm{Cr}_{0.5} \mathrm{Fe} 0.25\right)$ detected by XRD in the M2-CR-L sample sintered at $1000{ }^{\circ} \mathrm{C}$ may possibly surround the chromium particles as shown in Fig. 5f. It is more difficult to precisely locate the $\sigma$ phase in the M1cr sample with pre-milled chromium.

The EBSD phase map and the corresponding SEM image are shown in Fig. 7. Comparison between the two pictures demonstrates that the Al-Ni rich phase corresponds to the $\mathrm{BCC}$ phase observed in the diffraction patterns and the Fe-Cr-Co rich phase corresponds to the FCC phase. By combining the results of the Rietveld analysis, EBSD and SEM pictures, the volume fraction of each phase can be estimated. It is close to $50-50 \%$ and never exceeds $60 \%$ locally in the M2 milling samples. For M1 milling, the BCC phase constitutes over $75 \%$ of the sintered sample.
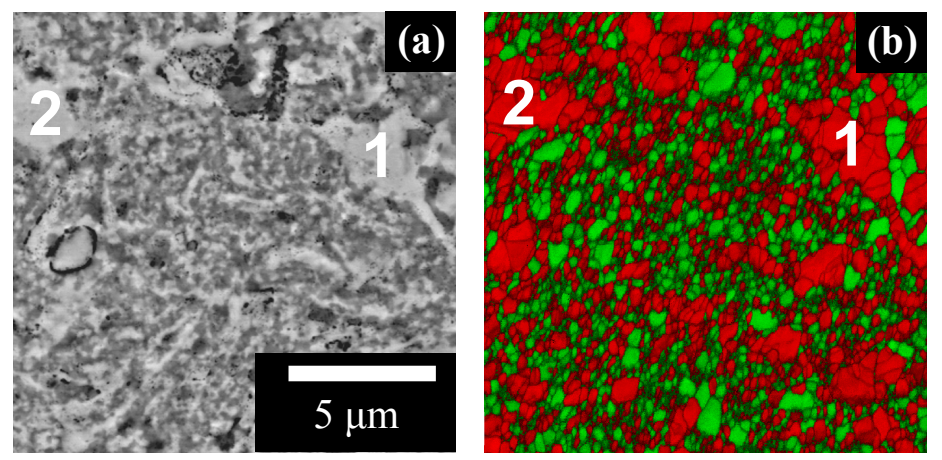

Figure 5: (a) SEM image (BSE) and (b) EBSD phase map of the $\mathrm{M} 2-$-milled, $1100^{\circ} \mathrm{C}$ sintered sample (green corresponding to $\mathrm{BCC}$ and red to $\mathrm{FCC}$ ).

In addition, XR Fluorescence results indicate that the composition of the whole M2-cr sintered sample is very close to equimolar. 
The CALPHAD approach was used in order to determine the FCC/BCC ratio at thermodynamic equilibrium. The phase volume fraction and corresponding composition are presented in Fig. $8 \mathrm{a}$ in the equimolar case $\mathrm{Al}_{\mathrm{x}} \mathrm{CoCrFeNi}$ $(\mathrm{x}=1)$. According to our calculations, based on the database TCNI9 [37] (Thermo-Calc [38]), only 2 BCC phases should form at the target temperatures $\left(1000^{\circ} \mathrm{C}\right.$ or $\left.1100^{\circ} \mathrm{C}\right)$. That is precisely observed in as-cast HEAs [3-7]. The BCC phase consists of an ordered and a disordered phase. In the case of the solid route used in this work, an FCC phase was also formed during elaboration. It does not seem that the presence of the FCC phase is due to the initial state of the milled agglomerates exhibiting the coexistence of FCC and $\mathrm{BCC}$ phases. Indeed, other groups report the formation of FCC and BCC phases by sintering from only BCC agglomerates [12].

Calculating with a slightly lower $\mathrm{Al}$ content, corresponding to $\mathrm{A} \mathrm{l}_{\mathrm{x}} \mathrm{CoCrFeNi}(\mathrm{x}=0.75)$ composition, gives the coexistence of a disordered FCC phase, $\mathrm{Al}_{6} \mathrm{Co}_{21} \mathrm{Cr}_{29} \mathrm{Fe}_{25} \mathrm{Ni}_{17}$, and an ordered $\mathrm{BCC}$ phase (Fig. 8b). The sigma phase is not expected in the range of processing temperatures. Although the agreement between thermodynamic calculations and experimental observations is encouraging, a quantitative discrepancy persists. Different assumptions may be formulated:

- The chosen composition $\mathrm{AlxCoCrFeNi}$ is at the border of two domains (Figs. 8a, 8b, 8c) and a slight shift in $\mathrm{Al}$ composition may induce different phase equilibriums.

- In our experiments, equilibrium has not been reached because of the solid-state fabrication. Indeed, powder metallurgy routes are known to produce metastable materials, especially with HEBM [39]. The effect of a heat treatment on the samples would be interesting way to detect the persistence of an FCC phase at higher temperatures.

- Chemical homogeneity is not reached locally: the fact that some areas are depleted of $\mathrm{Al}$ and reach an equilibrium corresponding to Fig. 8b could explain the presence of FCC. In addition, a slight decrease in Cr content could also lead to the formation of FCC (Fig. 8d).

- Finally, the database we use could be improved, especially since the $\mathrm{Al}_{1} \mathrm{CoCrFeNi}$ composition seems to be at the border of two domains. Indeed, other researchers come to different conclusions using different databases [40,41]. However, results similar to ours have been reported by Butler et al. [9].

We here report the material properties of the samples we fabricated. Relative density is $91.1 \%$ for M1-CR-L sample and 98\% for M2-CR-L. The M1-CR-L sample exhibits a hardness of $525 \mathrm{HV}$ and the M2-CR-L sample of $670 \mathrm{HV}$. Relative density is $97.5 \%$ for the M1-cr sample and 96\% for M2-cr. The sample sintered from the M1activated powder exhibits a hardness of $530 \mathrm{HV}$ and that from the M2-milled powder exhibits hardness of $610 \mathrm{HV}$. 
These values are consistent with the results from the literature. HEAs elaborated by the solid-state route are harder than arc-melted HEAs. For instance, an AlCoCrFeNi cast-alloy of $500 \mathrm{HV}$ was reported here [5] whereas the hardness of the same sintered alloy was found to attain $625 \mathrm{HV}$ [12]. Compared to cast alloys, increased hardness is mainly due to impurities resulting from the process (precipitation hardening, especially for hard ceramic precipitates) and limited grain size (grain boundary hardening) [32,42]. The M2-CR-L sample exhibits the highest hardness because of its highest content of sigma phase. Indeed, the sigma phase is known to increase hardness of similar alloys $[5,13]$.

It was not possible to measure the hardness of each phase separately with the micro-hardness instrument we used.

However, the difference in hardness between the M1 and M2 samples may be due to different $\mathrm{FCC} / \mathrm{BCC}$
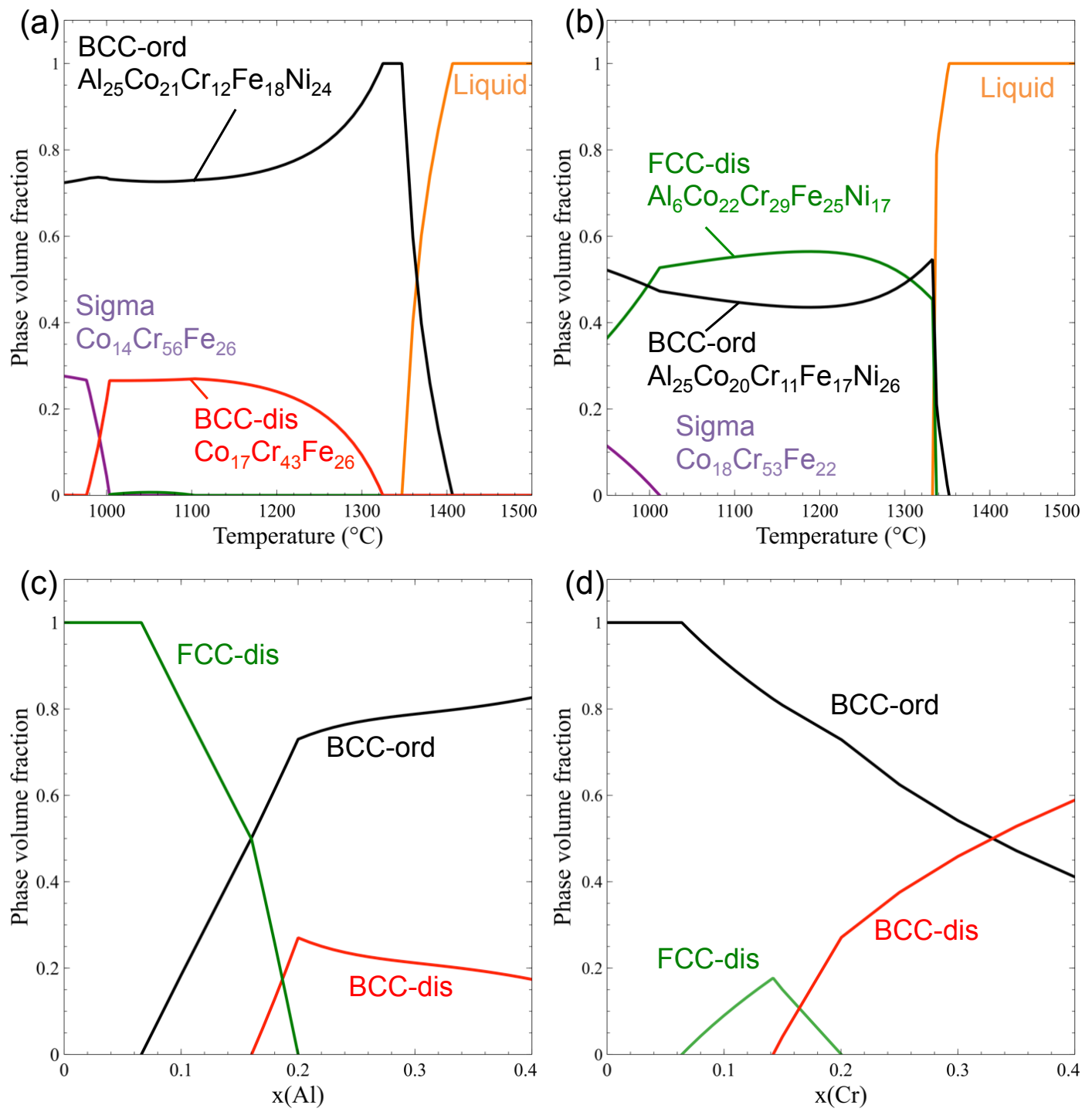

Figure 8: Phase volume fraction as a function of Temperature together with composition. $x(A l)=0.2$ $\mathrm{Al}_{1} \mathrm{CoCrFeNi}(\mathrm{a})$ and $\mathrm{x}(\mathrm{Al})=0.15 \mathrm{Al}_{0.75} \mathrm{CoCrFeNi}(\mathrm{b})$. Phase volume fraction as a function of Al content (c) and $\mathrm{Cr}$ content $(\mathrm{d})$ at sintering temperature $\left(1100^{\circ} \mathrm{C}\right)$. Thermo-Calc with TCNI9. 
distribution. Indeed, the Fe-Cr-Co rich FCC phase is more abundant in the M2 sample and could be harder than the Al-Ni rich BCC phase. 


\section{Conclusion}

In this work, we tested the versatility of powder metallurgy routes, especially by studying the combination of mechanical activation and reactive sintering. "Medium" energy ball milling of 5 elemental powders produced agglomerates of enhanced reactivity. Their microstructure depended on the milling conditions. The typical lamellar structure, observed in the $\mathrm{K}=1$ case, consists of closely imbricated elements. Complementary experimental characterization and numerical study of milled powders enabled us to accurately depict their crystalline state as precursors of solid solutions. Given the right parameters, the SPS process produced a nanostructured sample (M2cr) with two multicomponent solid solutions (BCC+FCC) homogenously distributed.

Our work, based on the reactive sintering of activated agglomerates, proves that a complete Mechanical Alloying is not necessary to elaborate High Entropy Alloys. We have demonstrated that this new way of producing HEAs leads to a unique microstructure. This kind of dual microstructure could have remarkable properties, especially mechanical ones [43]. For high temperature applications, the stability of this microstructure needs to be evaluated by long duration heat treatments. This study could also be valuably extended to other compositions and other metallic elements.

We have not yet identified, however, the nanosized precipitates formed during the process. They could be oxides (due to the impurities of the initial powders) or carbides (due to the use of graphite dies for SPS). Future work will thus need to determine the nature of these precipitates and find a way to hinder their formation.

It would also be interesting to correctly predict the amount of each phase via Calphad, either by using an improved database or by precisely understanding the reasons for the observed discrepancies. This improvement will enable us to tailor the mechanical properties of the high entropy alloys prepared by reactive sintering and to investigate other HEAs.

Finally, the milling and sintering steps can be further optimized to control the formation of unwanted phases $(\sigma)$ and impurities as well as to shorten the duration of the process. 


\section{Acknowledgments}

The use of computational facilities at the Computing Center of the University of Burgundy, PSIUN-CCUB, is gratefully acknowledged. We are grateful to Dr. Ludivine Minier for helpful discussions and expertise in Spark Plasma Sintering.

\section{References}

[1] J.-W. Yeh, S.-K. Chen, S.-J. Lin, J.-Y. Gan, T.-S. Chin, T.-T. Shun, C.-H. Tsau, S.-Y. Chang, Nanostructured High-Entropy Alloys with Multiple Principal Elements: Novel Alloy Design Concepts and Outcomes, Adv. Eng. Mater. 6 (2004) 299-303. doi:10.1002/adem.200300567.

[2] D.B. Miracle, O.N. Senkov, A critical review of high entropy alloys and related concepts, Acta Mater. 122 (2017) 448-511. doi:10.1016/j.actamat.2016.08.081.

[3] S. Uporov, V. Bykov, S. Pryanichnikov, A. Shubin, N. Uporova, Effect of synthesis route on structure and properties of AlCoCrFeNi high-entropy alloy, Intermetallics. 83 (2017) 1-8. doi:10.1016/j.intermet.2016.12.003.

[4] M. Ren, B. Li, H. Fu, Formation condition of solid solution type high-entropy alloy, Trans. Nonferrous Met. Soc. China. 23 (2013) 991-995. doi:10.1016/S1003-6326(13)62557-1.

[5] A. Munitz, S. Salhov, S. Hayun, N. Frage, Heat treatment impacts the micro-structure and mechanical properties of AlCoCrFeNi high entropy alloy, J. Alloys Compd. 683 (2016) 221-230. doi:10.1016/j.jallcom.2016.05.034.

[6] A. Manzoni, H. Daoud, R. Völkl, U. Glatzel, N. Wanderka, Phase separation in equiatomic AlCoCrFeNi high-entropy alloy, Ultramicroscopy. 132 (2013) 212-215. doi:10.1016/j.ultramic.2012.12.015.

[7] K.R. Lim, K.S. Lee, J.S. Lee, J.Y. Kim, H.J. Chang, Y.S. Na, Dual-phase high-entropy alloys for hightemperature structural applications, J. Alloys Compd. 728 (2017) 1235-1238. doi:10.1016/j.jallcom.2017.09.089.

[8] Y.P. Wang, B.S. Li, M.X. Ren, C. Yang, H.Z. Fu, Microstructure and compressive properties of AlCrFeCoNi high entropy alloy, Mater. Sci. Eng. A. 491 (2008) 154-158. doi:10.1016/j.msea.2008.01.064.

[9] T.M. Butler, M.L. Weaver, Oxidation behavior of arc melted AlCoCrFeNi multi-component high-entropy alloys, J. Alloys Compd. 674 (2016) 229-244. doi:10.1016/j.jallcom.2016.02.257.

[10] S. Varalakshmi, M. Kamaraj, B.S. Murty, Synthesis and characterization of nanocrystalline AlFeTiCrZnCu high entropy solid solution by mechanical alloying, J. Alloys Compd. 460 (2008) $253-257$. doi:10.1016/j.jallcom.2007.05.104.

[11] C. Suryanarayana, Mechanical alloying and milling, Prog. Mater. Sci. 46 (2001) 1-184. doi:10.1016/S0079-6425(99)00010-9.

[12] W. Ji, Z. Fu, W. Wang, H. Wang, J. Zhang, Y. Wang, F. Zhang, Mechanical alloying synthesis and spark plasma sintering consolidation of CoCrFeNiAl high-entropy alloy, J. Alloys Compd. 589 (2014) 61-66. doi:10.1016/j.jallcom.2013.11.146.

[13] S. Mohanty, T.N. Maity, S. Mukhopadhyay, S. Sarkar, N.P. Gurao, S. Bhowmick, K. Biswas, Powder metallurgical processing of equiatomic AlCoCrFeNi high entropy alloy: Microstructure and mechanical properties, Mater. Sci. Eng. A. 679 (2017) 299-313. doi:10.1016/j.msea.2016.09.062.

[14] C. Yang, J. Lin, J. Zeng, Shenggua. Qu, X. Li, W. Zhang, D. Zhang, High-Strength AlCrFeCoNi High Entropy Alloys Fabricated by Using Metallic Glass Powder as Precursor., Adv. Eng. Mater. 18 (2016) 348-353. doi:10.1002/adem.201500339.

[15] A. Zhang, J. Han, J. Meng, B. Su, P. Li, Rapid preparation of AlCoCrFeNi high entropy alloy by spark plasma sintering from elemental powder mixture, Mater. Lett. 181 (2016) 82-85. doi:10.1016/j.matlet.2016.06.014.

[16] M. Vaidya, A. Prasad, A. Parakh, B.S. Murty, Influence of sequence of elemental addition on phase evolution in nanocrystalline AlCoCrFeNi: Novel approach to alloy synthesis using mechanical alloying, Mater. Des. 126 (2017) 37-46. doi:10.1016/j.matdes.2017.04.027.

[17] E. Colombini, R. Rosa, L. Trombi, M. Zadra, A. Casagrande, P. Veronesi, High entropy alloys obtained 
by field assisted powder metallurgy route: SPS and microwave heating, Mater. Chem. Phys. (2017).

[18] E. Gaffet, F. Bernard, J.-C. Niepce, F. Charlot, C. Gras, G. Le Caër, J.-L. Guichard, P. Delcroix, A. Mocellin, O. Tillement, Some recent developments in mechanical activation and mechanosynthesis, J. Mater. Chem. 9 (1999) 305-314. doi:10.1039/a804645j.

[17] S. Plimpton, Fast Parallel Algorithms for Short-Range Molecular Dynamics, J. Comput. Phys. 117 (1995) 42. doi:10.1006/jeph.1995.1039. http://lammps.sandia.gov.

[20] X.W. Zhou, R.A. Johnson, H.N.G. Wadley, Misfit-energy-increasing dislocations in vapor-deposited CoFe/NiFe multilayers, Phys. Rev. B. 69 (2004). doi:10.1103/PhysRevB.69.144113.

[21] Z. Lin, R.A. Johnson, L.V. Zhigilei, Computational study of the generation of crystal defects in a bcc metal target irradiated by short laser pulses, Phys. Rev. B. 77 (2008). doi:10.1103/PhysRevB.77.214108.

[22] R.A. Johnson, Alloy models with the embedded-atom method, Phys. Rev. B. 39 (1989) 12554-12559. doi:10.1103/PhysRevB.39.12554.

[23] S. Odunuga, Y. Li, P. Krasnochtchekov, P. Bellon, R.S. Averback, Forced Chemical Mixing in Alloys Driven by Plastic Deformation, Phys. Rev. Lett. 95 (2005). doi:10.1103/PhysRevLett.95.045901.

[24] A. Perron, S. Garruchet, O. Politano, G. Aral, V. Vignal, Oxidation of nanocrystalline aluminum by variable charge molecular dynamics, J. Phys. Chem. Solids. 71 (2010) 119-124. doi:10.1016/j.jpcs.2009.09.008.

[25] A. Stukowski, Structure identification methods for atomistic simulations of crystalline materials, Model. Simul. Mater. Sci. Eng. 20 (2012) 045021. doi:10.1088/0965-0393/20/4/045021.

[26] J.H. Li, L.T. Kong, B.X. Liu, Proposed Definition of Microchemical Inhomogeneity and Application To Characterize Some Selected Miscible/Immiscible Binary Metal Systems, J. Phys. Chem. B. 108 (2004) 1607116076. doi:10.1021/jp047897x.

[27] A.F. Wells, Structural Inorganic Chemistry, 5th ed., Clarendon Press, Oxford, 1984.

[28] J. Wallenius, P. Olsson, C. Lagerstedt, N. Sandberg, R. Chakarova, V. Pontikis, Modeling of chromium precipitation in Fe-Cr alloys, Phys. Rev. B. 69 (2004). doi:10.1103/PhysRevB.69.094103.

[29] V.K. Portnoi, A.V. Leonov, S.E. Filippova, A.N. Streletskii, A.I. Logacheva, Mechanochemical synthesis and heating-induced transformations of a high-entropy Cr-Fe-Co-Ni-Al-Ti alloy, Inorg. Mater. 50 (2014) 1202 1211. doi:10.1134/S0020168514120188.

[30] N. Burgio, A. Iasonna, M. Magini, S. Martelli, F. Padella, Mechanical alloying of the $\mathrm{Fe}-\mathrm{Zr}$ system. Correlation between input energy and end products, Il Nuovo Cimento D. 13 (1991) 459-476. doi:10.1007/BF02452130.

[31] M. Abdellaoui, E. Gaffet, The physics of mechanical alloying in a planetary ball mill: Mathematical treatment, Acta Metall. Mater. 43 (1995) 1087-1098. doi:10.1016/0956-7151(95)92625-7.

[32] I. Moravcik, J. Cizek, P. Gavendova, S. Sheikh, S. Guo, I. Dlouhy, Effect of heat treatment on microstructure and mechanical properties of spark plasma sintered AlCoCrFeNiTi0.5 high entropy alloy, Mater. Lett. 174 (2016) 53-56. doi:10.1016/j.matlet.2016.03.077.

[33] A.S. Rogachev, D.O. Moskovskikh, A.A. Nepapushev, T.A. Sviridova, S.G. Vadchenko, S.A. Rogachev, A.S. Mukasyan, Experimental investigation of milling regimes in planetary ball mill and their influence on structure and reactivity of gasless powder exothermic mixtures, Powder Technol. 274 (2015) 44-52. doi:10.1016/j.powtec.2015.01.009.

[34] S. Rosenkranz, S. Breitung-Faes, A. Kwade, Experimental investigations and modelling of the ball motion in planetary ball mills, Powder Technol. 212 (2011) 224-230. doi:10.1016/j.powtec.2011.05.021.

[35] J. Cieslak, J. Tobola, K. Berent, M. Marciszko, Phase composition of Al x FeNiCrCo high entropy alloys prepared by sintering and arc-melting methods, J. Alloys Compd. 740 (2018) 264-272. doi:10.1016/j.jallcom.2017.12.333.

[36] S.-H. Joo, H. Kato, M.J. Jang, J. Moon, E.B. Kim, S.-J. Hong, H.S. Kim, Structure and properties of ultrafine-grained $\mathrm{CoCrFeMnNi}$ high-entropy alloys produced by mechanical alloying and spark plasma sintering, J. Alloys Compd. 698 (2017) 591-604. doi:10.1016/j.jallcom.2016.12.010.

[36] TCNI9: Thermo-Calc Ni-base alloys database, version 9.0. Developed and provided by Thermo-Calc Software AB (2019), https://www.thermocalc.com/media/83726/TCNI9_extended_info.pdf

[38] J.-O. Andersson, T. Helander, L. Höglund, P. Shi, B. Sundman, Thermo-Călc \& DICTRA, computational tools for materials science, Calphad. 26 (2002) 273-312. doi:10.1016/S0364-5916(02)00037-8.

[39] C.C. Koch, O.B. Cavin, C.G. McKamey, J.O. Scarbrough, Preparation of "amorphous" Ni $60 \mathrm{Nb} 40$ by mechanical alloying, Appl. Phys. Lett. 43 (1983) 1017-1019. doi:10.1063/1.94213.

[40] C. Zhang, F. Zhang, H. Diao, M.C. Gao, Z. Tang, J.D. Poplawsky, P.K. Liaw, Understanding phase stability of Al-Co-Cr-Fe-Ni high entropy alloys, Mater. Des. 109 (2016) 425-433. doi:10.1016/j.matdes.2016.07.073.

[41] A. Manzoni, S. Singh, H. Daoud, R. Popp, R. Völkl, U. Glatzel, N. Wanderka, On the Path to Optimizing the Al-Co-Cr-Cu-Fe-Ni-Ti High Entropy Alloy Family for High Temperature Applications, Entropy. 18 (2016) 104. doi:10.3390/e18040104.

[42] C. Shang, E. Axinte, J. Sun, X. Li, P. Li, J. Du, P. Qiao, Y. Wang, CoCrFeNi(W 1-x Mo x ) high-entropy 
alloy coatings with excellent mechanical properties and corrosion resistance prepared by mechanical alloying and hot pressing sintering, Mater. Des. 117 (2017) 193-202. doi:10.1016/j.matdes.2016.12.076.

[43] S. Gangireddy, B. Gwalani, V. Soni, R. Banerjee, R.S. Mishra, Contrasting mechanical behavior in precipitation hardenable AlXCoCrFeNi high entropy alloy microstructures: Single phase FCC vs. dual phase FCCBCC, Mater. Sci. Eng. A. 739 (2019) 158-166. doi:10.1016/j.msea.2018.10.021.

\section{Supplementary data}
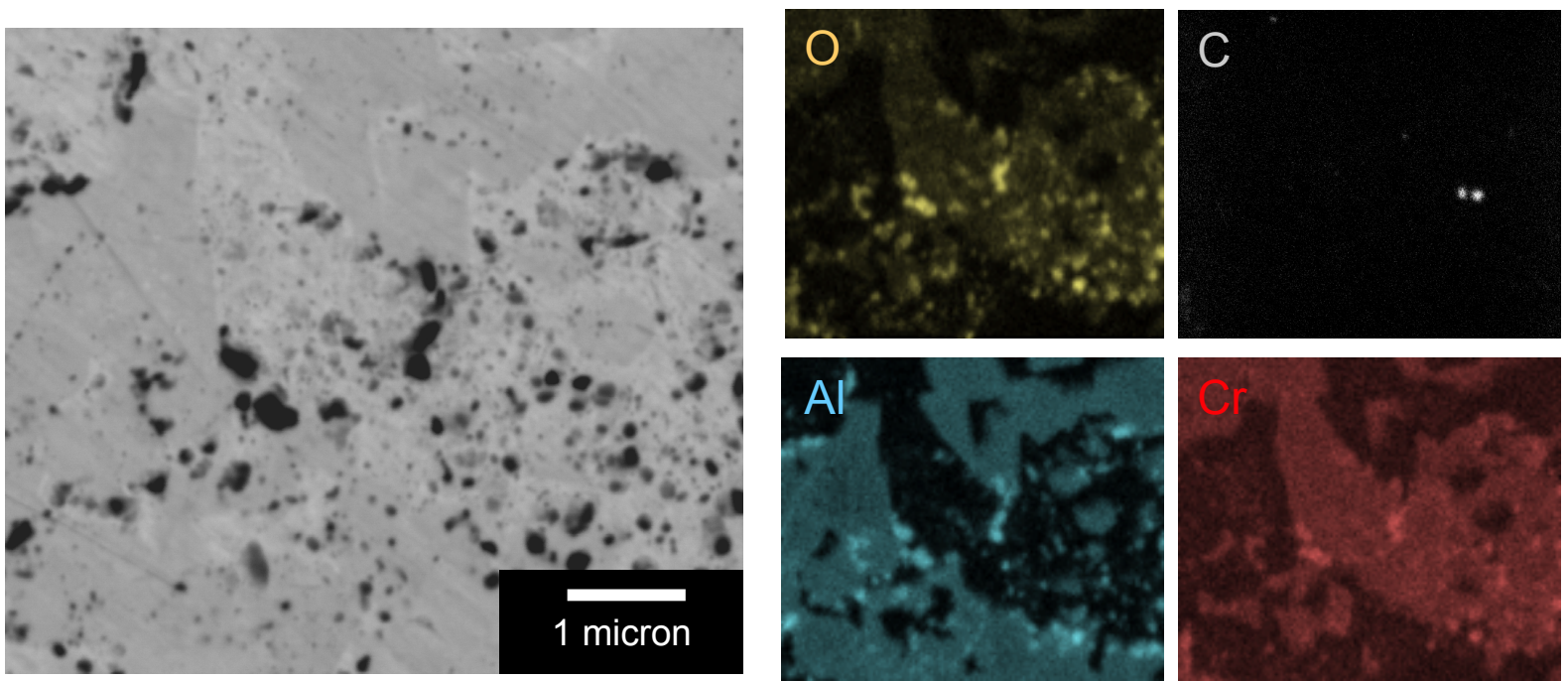

Figure A1 : SEM image (BSE mode). O, C, Al and Cr distribution of the sample M1-cr sintered at $1100^{\circ} \mathrm{C}$. 